05,09

\title{
Особенности магниторефрактивного эффекта в многослойной металлической наноструктуре $[\mathrm{CoFe} / \mathrm{Cu}]_{n}{ }^{*}$
}

\author{
() А.Н. Юрасов ${ }^{1}$, А.В. Телегин², Н.С. Банникова², М.А. Миляев², Ю.П. Сухоруков² \\ ${ }^{1}$ Московский технологический университет, \\ Москва, Россия \\ ${ }^{2}$ Институт ффизики металлов им. М.Н. Михеева УрО РАН, \\ Екатеринбург, Россия \\ E-mail: alexey_yurasov@mail.ru
}

(Поступила в Редакцию 22 июня 2017 г.)

\begin{abstract}
Теоретически и экспериментально изучены особенности магниторефрактивного эффекта в металлических многослойных пленочных наноструктурах $\mathrm{Ni}_{48} \mathrm{Fe}_{12} \mathrm{Cr}_{40}(50 \AA) /\left[\mathrm{Cog}_{90} \mathrm{Fe}_{10}(14 \AA) / \mathrm{Cu}(22 \AA)\right]_{8} / \mathrm{Cr}(20 \AA)$, обладающих эффектом гигантского магнитосопротивления при комнатных температурах. Показано, что магниторефрактивный эффект в таких структурах достигает $1.5 \%$ в магнитном поле $3.5 \mathrm{kOe}$ в широкой ИК-области спектра и может менять знак как на пропускании, так и на отражении света. Рассчитаны величины коэффициентов рефракции и экстинкции для наноструктур в магнитном поле, которые хорошо согласуются с полученными экспериментальными данными. Полученные формулы можно использовать для оценки магниторефрактивного эффекта в металлических многослойных структурах.
\end{abstract}

Работа выполнена в рамках государственного задания ФАНО России (тема „Спин“ № 01201463330), при частичной поддержке программы УрО РАН № 18-10-2-4 и гранта Министерства образования и науки РФ № 14.Z50.31.0025 и гранта Президента РФ для ведущих научных школ НШ-8003.2016.2.

DOI: $10.21883 /$ FTT.2018.02.45381.201

\section{1. Введение}

Обнаружение и изучение в металлических структуpax и в манганитах перспективных для практического применения эффектов, связанных с изменением электросопротивления на постоянном токе в магнитном поле, - гигантского и колоссального магнитосопротивления (ГМС и КМС соответственно) - стимулировало также поиск высокочастотного (оптического) отклика на эти эффекты, рассматриваемого как изменение коэффициента отражения $(R)$ и пропускания $(T)$ электромагнитного излучения под действием магнитного поля - магнитоотражение и магнитопропускание света, соответственно (см. [1-4] и ссылки в них). Оптический отклик наиболее ярко проявился в области взаимодействия света с носителями заряда при частотах $\omega \leq \omega_{p}$ $\left(\omega_{p}-\right.$ плазменная частота $)$, т. е. для металлов и магнитных полупроводников - в инфракрасной (ИК) области спектра (например, [5-7] и ссылки в них). Появление экспериментальных результатов позволило разработать теорию магниторефрактивного эффекта (МРЭ), преимущественно в области $\omega \tau<1(\tau-$ время релаксации), для металлов $[5,9]$, гранулированных и нанокомпозитных металлических структур [10,11], тонкопленочных наноструктур с ГМС [12] и манганитов с КМС [13]. Важным фактом является то, что согласно экспериментальным данным и теоретическим оценкам МРЭ на

\footnotetext{
* Доклад на XX Международном симпозиуме „Нанофизика и наноэлектроника“, Н. Новгород, 13-16 марта 2017 г.

Материалы симпозиума частично опубликованы в выпуске № 11 за 2017 г. журнала „Физика твердого тела“.
}

отражении и пропускании естественного (неполяризованного) света величина эффекта в ИК-области спектра существенно превышает по интенсивности величины традиционных магнитооптических эффектов в поляризованном свете [13]. Вместе с тем в каждом случае существуют особенности МРЭ, связанные с зарядовыми и структурными свойствами вещества, которые требуют развития моделей МРЭ для каждого класса магнитных материалов и/или спектральной области исследования. Например, учет вклада межзонных переходов в металлических структурах [14], фазового расслоения в случае легированных манганитов с КМС [15], интерференции и поглощения света в случае многослойных структур [12] и т.д. Таким образом, несмотря на большое количество теоретических и экспериментальных работ по МРЭ в металлических системах, представляет интерес анализ механизмов МРЭ в тонкопленочных наноструктурах в сопоставлении с экспериментальными данными с целью усовершенствования теории и развития методов бесконтактного контроля качества и оценки величины ГМС таких структур.

Данная работа посвящена теоретическому и экспериментальному изучению МРЭ в пленочной многослойной наноструктуре на примере системы $\mathrm{Ni}_{48} \mathrm{Fe}_{12} \mathrm{Cr}_{40}(50 \AA) /$ $\left[\mathrm{Co}_{90} \mathrm{Fe}_{10}(14 \AA) / \mathrm{Cu}(22 \AA)\right]_{8} / \mathrm{Cr}(20 \AA)$, обладающей эффектом ГМС в слабых магнитных полях $(H<1 \mathrm{kOe})$ при комнатной температуре. Получены формулы, описывающие изменение коэффициентов рефракции и экстинкции в магнитном поле. Проведены расчета МРЭ в моделях эффективной среды с использованием формул Френеля, а также с учетом спин-зависящего рассеяния электронов в пределе самоусреднения. 


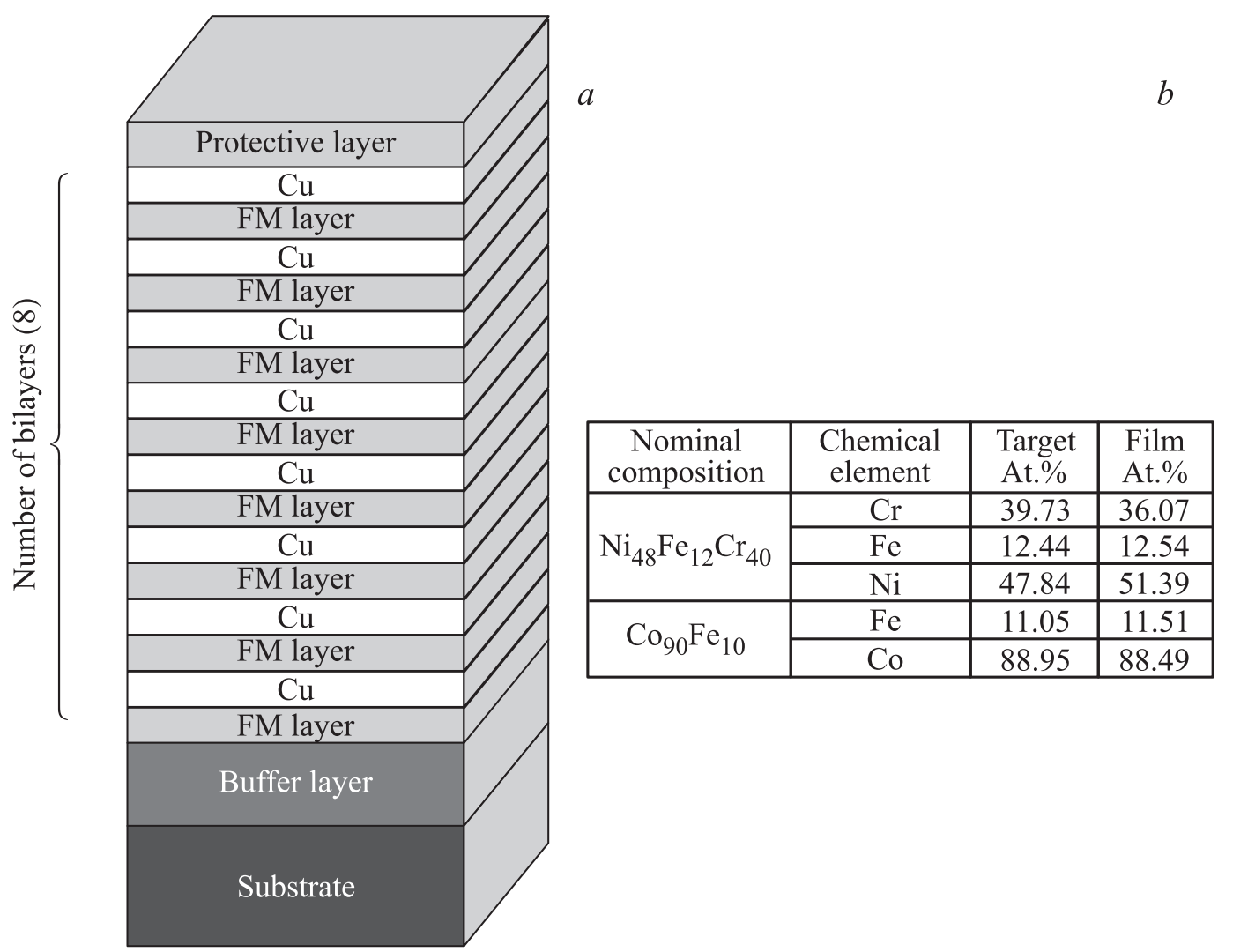

Рис. 1. $a$ - схематическое изображение многослойной структуры $\mathrm{Ni}_{48} \mathrm{Fe}_{12} \mathrm{Cr}_{40} /\left[\mathrm{Co}{ }_{90} \mathrm{Fe}_{10} / \mathrm{Cu}\right]_{8} / \mathrm{Cr}, b-$ элементный состав сплавов в исходных мишенях и напыленных калибровочных пленках.

\section{2. Образцы и техника эксперимента}

Наноструктуры $\quad \mathrm{Ni}_{48} \mathrm{Fe}_{12} \mathrm{Cr}_{40}(50 \AA) /\left[\mathrm{Co}_{90} \mathrm{Fe}_{10}(14 \AA) /\right.$ $\mathrm{Cu}(22 \AA)]_{8} / \mathrm{Cr}(20 \AA)$ были выращены на стеклянной подложке (аморфный $\mathrm{SiO}_{2}$ толщиной $0.46 \mathrm{~mm}$ ) методом высоковакуумного магнетронного напыления на постоянном токе при комнатной температуре, детально описанном в работе [16]. Восемь пар слоев структуры (рис. $1, a$ ) напылялись из мишеней соответствующего состава. Элементный состав слоев определялся на энергодисперсионном рентгеновском спектрометре GENESIS APEX 2 EDS (рис. 1,b). Первый слой $\mathrm{Ni}_{48} \mathrm{Fe}_{12} \mathrm{Cr}_{40}$ является буферным слоем для сглаживания шероховатости подложки $\mathrm{SiO}_{2}$. В наноструктурах с буферным слоем $\mathrm{NiFeCr}$ формируется совершенная кристаллическая структура слоев и область существования острой аксиальной текстуры с осью $\langle 111\rangle[16]$. Слои $\mathrm{Cu}$ обеспечивают обменное взаимодействие антиферромагнитного типа между слоями $\mathrm{CoFe}$, а верхний слой $\mathrm{Cr}(20 \AA)$ предназначен для защиты наноструктуры от окисления. Число пар и толщина слоев выбирались, исходя из требований максимального ГМС при сохранении полупрозрачности образца для наблюдения магнитооптического отклика как на отражение, так и на пропускание света.

Электросопротивление и магнитосопротивление образцов размером $2 \times 8 \mathrm{~mm}$ измерялись на постоянном токе 4-х контактным методом. Магнитосопротивление в работе определялось как $\Delta \rho / \rho_{0}=\left(\rho_{H}-\rho_{0}\right) / \rho_{0} \cdot 100 \%$, где $\rho_{H}$ - электросопротивление образца во внешнем магнитном поле величиной до $H=15 \mathrm{kOe}, \rho_{0}-$ электросопротивление без поля. Вектор напряженности магнитного поля и постоянный ток были направлены взаимно перпендикулярно в плоскости слоев пленки (CIP геометрия). Пропускание $(T)$ и коэффициент отражения $(R)$ неполяризованного света измерялись при нормальном падении света к поверхности образца (угол падения $\sim 7^{\circ}$ ) в диапазоне длин волн $1-11 \mu \mathrm{m}$ при $T=295 \mathrm{~K}$. Влияние магнитного поля на пропускание и отражение света исследовалось в полях до $8 \mathrm{kOe}$ в плоскости пленки (геометрия Фохта). Магниторефрактивный эффект на пропускание и отражение света определялся через экспериментально наблюдаемые магнитопропускание $(\Delta T / T)$ и магнитоотражение $(\Delta R / R)$ света, рассчитываемые соответственно по формулам

$$
\Delta T / T=\left(T_{H}-T_{0}\right) / T_{0} \text { и } \Delta R / R=\left(R_{H}-R_{0}\right) / R_{0},
$$

где $T_{H, 0}$ и $R_{H, 0}$ - интенсивности прошедшего или отраженного света во внешнем магнитном поле и без поля соответственно. Погрешность измерения $\Delta T / T$ и $\Delta R / R$ в наноструктуре составила $\sim 0.3$ и 0.1 соответственно. 


\section{3. Анализ особенностей МРЭ в многослойных наноструктурах}

Известно, что в оптической области спектра реакция электронной системы металла на электромагнитное поле световой волны определяется в основном тензором диэлектрической проницаемости $\hat{\varepsilon}(\omega, k)$. Пространственная дисперсия $\hat{\varepsilon}(k)$ в металлах и их сплавах в оптическом диапазоне спектра незначительна, и ей можно пренебречь. Поэтому в отсутствие магнитного поля $\varepsilon(\omega)=\varepsilon_{1}(\omega)-i \varepsilon_{2}(\omega)-$ скалярная величина, действительная и мнимая части которой определяются через индексы рефракции $n$ и $k$

$$
\operatorname{Re} \varepsilon(\omega)=\varepsilon_{1}(\omega)=n^{2}-k^{2}, \operatorname{Im} \varepsilon(\omega)=\varepsilon_{2}(\omega)=2 n k .
$$

Оптическая проводимость металла определяется как

$$
\sigma(\omega)=\frac{\omega n k}{2 \pi}=\frac{\omega \operatorname{Im} \varepsilon(\omega)}{4 \pi}
$$

Отклик намагниченной среды на действие электрического поля световой волны сводится к появлению в тензоре $\hat{\varepsilon}$ отличной от нуля недиагональной компоненты $\varepsilon^{\prime}(\omega)=\varepsilon_{1}^{\prime}(\omega)-i \varepsilon_{2}^{\prime}(\omega)$, а строящийся на основе вынужденной анизотропии тензор $\hat{\varepsilon}$ ферромагнетика показывает, что намагниченная среда в оптическом отношении может рассматриваться как двупреломляющий (гиротропный) кристалл. Все магнитооптические эффекты изменение интенсивности, фазы и состояния поляризации излучения при его взаимодействии с намагниченным или магнитоупорядоченным веществом - обусловлены возникновением кругового или линейного двупреломления и дихроизма в намагниченной среде.

В работах $[11,13,17]$ было отмечено, что в ИК-области спектра вклад недиагональных компонент тензора диэлектрической проницаемости для магнитного металла не существен, и наблюдаемые изменения в магнитном поле определяются только вкладом диагональных компонент среды из магнитного и немагнитного металла. Следовательно, вклад линейных и нелинейных по полю магнитооптических эффектов в МРЭ в ИК-диапазоне будет минимальным. Таким образом, в геометрии нормального падения неполяризованного ИК-излучения в намагниченных металлических многослойных пленочных наноструктурах будет анализироваться только МРЭ, связанный с диагональной частью тензора диэлектрической проницаемости.

В многослойных металлических наноструктурах проводимость $\sigma(\omega)$ образца существенно изменяется при намагничивании и поэтому диэлектрическая проницаемость $\varepsilon(\omega)$

$$
\varepsilon(\omega)=\varepsilon_{r}(\omega)-i \frac{4 \pi \sigma(\omega)}{\omega}
$$

линейно связанная с проводимостью $\sigma(\omega)$ всей структуры, также является функцией приложенного магнитного поля, где $\omega$ - частота электромагнитного излучения, $\varepsilon_{r}(\omega)$ - диэлектрическая проницаемость, учитывающая вклад токов смещения. Согласно [12] МРЭ может быть описан как изменение коэффициентов отражения и пропускания электромагнитных волн для образцов с магниторезистивным эффектом (ГМС, КМС и т.д.) при их намагничивании. Эффект проявляется наиболее ярко в ИК-области спектра, где внутризонная динамика электронов проводимости играет доминирующую роль. Вклад намагниченности образца $(M)$ в его оптическую проводимость $\sigma(\omega)$ и соответственно в МРЭ учитывается посредством ее влияния на магнитосопротивление $\Delta \rho / \rho_{0}$ структуры [11]

$$
\Delta \sigma=\frac{1}{\rho_{0}}-\frac{1}{\rho_{H}}=\frac{\Delta \rho}{\rho_{0}^{2}\left[1-\frac{\Delta \rho}{\rho_{0}}\right]},
$$

где $\Delta \sigma-$ магнитопроводимость структуры, $\Delta \rho=$ $=\rho_{H}-\rho_{0}$. Нижние индексы $H$ и 0 дают значения указанных величин во внешнем магнитном поле и без поля.

Важной задачей при расчете МРЭ является правильный расчет коэффициента отражения и пропускания света в магнитном поле. Для этого в выражениях (2) в магнитном поле коэффициент рефракции $n$ и коэффициент экстинкции $k$ заменяются на $n_{H}=n+\Delta n$ и $k_{H}=k+\Delta k$. В формуле (4) выделяется действительная и мнимая части, $\varepsilon_{1}$ и $\varepsilon_{2}$. Приравняв $n_{H}$ и $k_{H}$ к соответствующим выражениям из формулы (4) и пренебрегая произведением $\Delta n \Delta k$ как величиной второго порядка малости, окончательно получим формулы для $\Delta n$ и $\Delta k$ и их связь с изменением магнитопроводимости, а значит, и с магнитосопротивлением наноструктуры по формуле

$$
\begin{aligned}
& \Delta n=\frac{2 \pi \Delta \sigma}{\omega}\left(\frac{n+k}{n^{2}+k^{2}}\right), \\
& \Delta k=\frac{2 \pi \Delta \sigma}{\omega}\left(\frac{n-k}{n^{2}+k^{2}}\right) .
\end{aligned}
$$

Таким образом, главный вклад в изменение коэффициентов прямо пропорционален магнитопроводимости структуры. Полученные формулы позволяют эффективно и просто оценивать МРЭ в любых наноструктурах через оптические параметры и магнитопроводимость (магнитосопротивление) образца.

Как правило, для расчета МРЭ в многослойной структуре последнюю в пределе самоусреднения представляют виде эффективной среды и далее используют формулы Френеля как решения уравнений Максвелла для распространения электромагнитных волн в эффективной трехслойной среде (вакуум-металлическая пленкаподложка) [18]. Формулы Френеля для коэффициента отражения $R$ трехслойной системы в случае нормального падения света имеют вид

$$
\begin{gathered}
R=\left|r_{j k l}^{p}\right|^{2}, r_{j k l}^{p}=\frac{r_{j k}^{p}+F_{k}^{2} r_{k l}^{p}}{1+F_{k}^{2} r_{j k}^{p} r_{k l}^{p}}, r_{j k}^{p}=\frac{g_{j} n_{k}^{2}-g_{k} n_{j}^{2}}{g_{j} n_{k}^{2}+g_{k} n_{j}^{2}}, \\
g_{j k l}=\sqrt{n_{j k l}^{2}-n_{1}^{2} \sin ^{2} \phi}, \quad F_{k}=\exp \left(-2 \pi i \lambda^{-1} g_{k} d_{k}\right),
\end{gathered}
$$

$\phi$ - угол падения света из первого слоя; $j, k, l-$ порядковые номера слоев; $d_{k}$ - толщина соответству- 
ющего слоя; $\lambda$ - длина световой волны в вакууме; $i$ - мнимая единица; $r$ - парциальный коэффициент отражения в отдельном слое; $F_{k}-$ фазовый множитель, ответственный за интерференцию и затухание излучения в $k$-слое структуры с толщиной $d$.

Аналогично данному подходу, можно рассчитать и пропускание света $T$ трехслойной системы [18]

$$
T=\left|t_{123}\right|^{2}, \quad t_{123}=\frac{t_{12} F_{2} t_{23}}{1+F_{2}^{2} r_{12} r_{23}} .
$$

Здесь и далее учитывается, что для нормального падения поляризационные компоненты естественного света равны: $r_{j k l}^{p}=-r_{j k l}^{s}=r_{j k l}$ и $t_{j k l}^{p}=t_{j k l}^{s}=t_{j k l}$.

В расчетах (7) в качестве индексов рефракции слоев без магнитного поля использовались известные табличные данные. При этом параметры $n$ и $k$ для эффективного металлического слоя $(d \approx 30 \mathrm{~nm})$ рассчитывались в рамках теории эффективной среды в приближении Бруггемана с учетом объемных концентраций компонент металлической структуры из $n$ - и $k$-металлов составляющих слоев наноструктуры подобно [17]. В самом первом приближении данные величины можно оценить как среднеарифметические взвешенные. Полученные значения коррелируют с характерными значениями для металлических сплавов.

Так как формулы Френеля являются точным решением уравнений Максвелла с учетом интерференции и поглощения света в металлических слоях многослойной структуры для более точной оценки МРЭ разумным подходом является учет параметров каждого слоя. При этом используются рекуррентные формулы Френеля для многослойных структур [18]

$$
\begin{gathered}
R=\left|r_{m, n+1}\right|^{2}, r_{m, n+1}=\frac{r_{m, m+1}+F_{m+1}^{2} r_{m+1, n+1}}{1+F_{m+1}^{2} r_{m, m+1} r_{m+1, n+1}} \\
r_{m, m+1}=\frac{g_{m} n_{m+1}^{2}-g_{m+1} n_{m}^{2}}{g_{m} n_{m+1}^{2}+g_{m+1} n_{m}^{2}}, g_{m}=\sqrt{n_{m}^{2}-n_{1}^{2} \sin ^{2} \phi} \\
F_{m+1}=\exp \left(-2 \pi i \lambda^{-1} g_{m+1} d_{m+1}\right),
\end{gathered}
$$

где $m$ - номер слоя, из которого падает свет, $n+1-$ номер слоя, в который свет выходит из образца (из всей многослойной структуры).

Аналогично рассчитывается пропускание света $T$

$$
T=\left|t_{m, n+1}\right|^{2}, \quad t_{m, n+1}=\frac{t_{m, m+1} F_{m+1} t_{m+1, n+1}}{1+F_{m+1}^{2} r_{m, m+1} r_{m+1, n+1}} .
$$

Отметим, что данный подход обычно используют для анализа оптических свойств многослойных структур с толщинами слоев близкой или большей длины волны излучения. Строго говоря, в случае ИК-спектроскопии структуры с наноразмерной толщиной слоев данное рассмотрение является избыточным.

Однако с учетом сильного поглощения света даже в тонких металлических наноструктурах, неидеальности границ раздела сред, конечного перемешивания слоев,
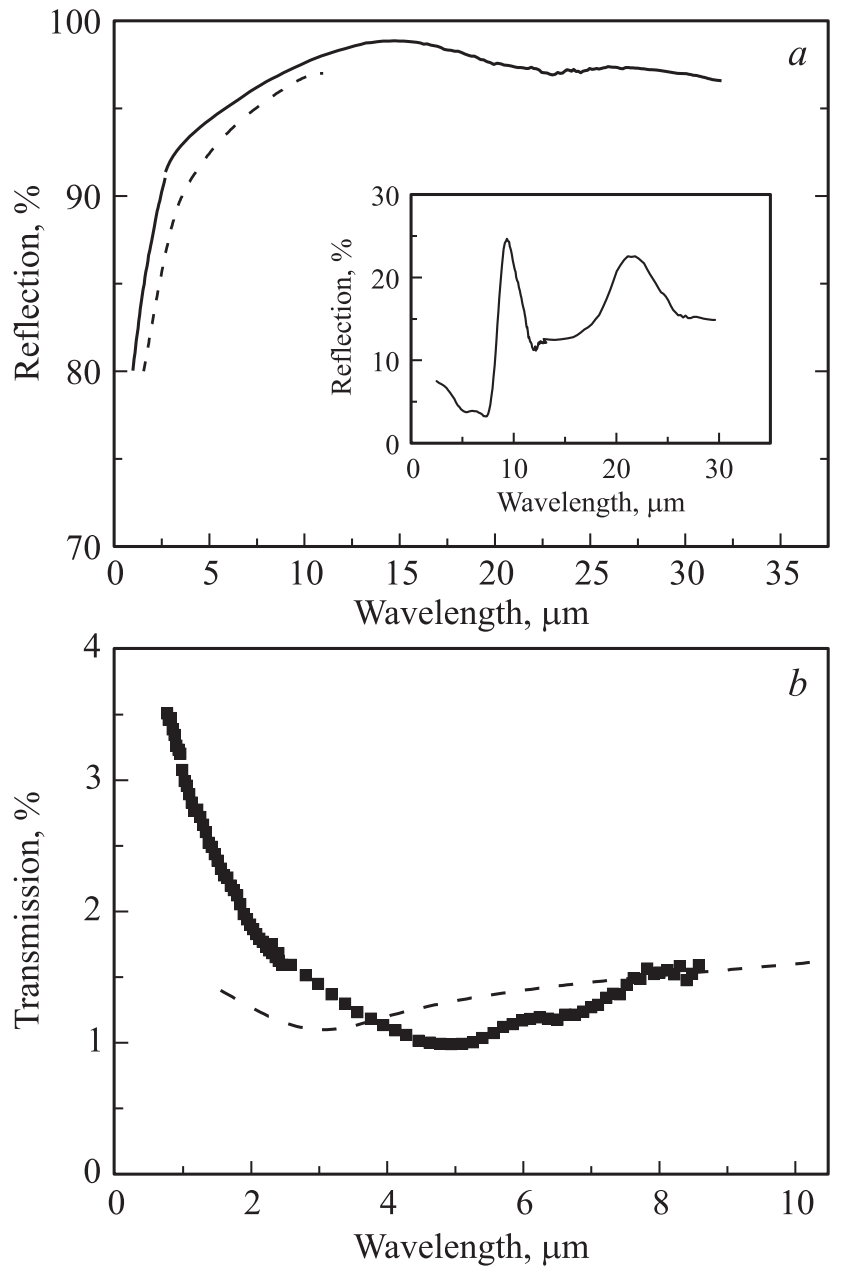

Рис. 2. $a-$ спектр зеркального отражения наноструктуры: штриховая линия - расчет в рамках модели эффективной среды, сплошная линия - эксперимент. На вставке - спектр отражения подложки $\mathrm{SiO}_{2} ; b-$ спектры пропускания наноструктуры: штриховая линия - расчет в рамках модели эффективной среды, сплошная линия - эксперимент.

а также размерных эффектов в случае наличия неоднородностей в структурах и возможности существования различных резонансных эффектов представляет интерес сравнительное изучение двух подходов в сопоставлении с прямыми экспериментальными данными.

Расчетные данные по формулам (7), (8) практически совпадают и демонстрируют хорошее согласие с измеренным коэффициентом отражения (рис. 2) и удовлетворительное - с пропусканием многослойной структуры (не показано), что, возможно, связано с сильным вкладом интерфейсных эффектов и спин-зависящего рассеяния электронов, которое также необходимо учесть. Вклад подложки в отражении света маскируется сильным поглощением в слоях структуры. Так как расчет для эффективно-трехслойной и многослойной структур дал близкие результаты, на рис. 2 приведены данные только для рассчетов по формулам (7), которые далее будут использованы для расчета МРЭ. 
Хотя расчеты по формулам Френеля позволяют учитывать интерференционные эффекты и поглощение в структуре, в них в явном виде не учитывается спинзависящее рассеяние носителей, являющееся основным механизмом магнитосопротивления в многослойных наноструктурах. Для учета спин-зависящего рассеяния использовалось известное выражение для диэлектрической проницаемости в пределе самоусреднения [19]

$$
\varepsilon=\varepsilon_{s t}+\left(\frac{\omega_{p}}{\omega}\right)^{2} \frac{i \omega \tau}{1-i \omega \tau}\left(1+\frac{m^{2} \beta^{2}}{(1-i \omega \tau)^{2}-m^{2} \beta^{2}}\right),
$$

где $m$ - относительная величина намагниченности системы $M / M_{s}, \beta$ - параметр спиновой асимметрии.

Модельные параметры варьировались таким образом, чтобы они соответствовали имеющимся экспериментальным оптическим данным. Так, было взято $\varepsilon_{s t}=3.5$, что соответствует модифицированной модели Друде для соединений такого типа в ИК-области спектра [19]. Параметры $m$ и $\beta$ подбирались как для единой эффективной металлической среды и составили $\tau=9 \cdot 10^{-16} \mathrm{~s}$, $\beta=0.75$, как в [19]. Данные значения позволили получить спектры МРЭ максимально близкие к экспериментальным и не противоречат результатам для аналогичных структур [20]. Влияние внешнего магнитного поля учитывалось за счет члена $m$. Далее рассчитывались коэффициенты отражения и пропускания света в поле и без поля и затем по формуле (1) рассчитывались магнитопропускание и магнитоотражение света с учетом спин-зависящего рассеяния в наноструктуре.

Результаты расчета МРЭ в многослойной металлической наноструктуре $\mathrm{Ni}_{48} \mathrm{Fe}_{12} \mathrm{Cr}_{40}(50 \AA) /\left[\mathrm{Co}_{90} \mathrm{Fe}_{10}(14 \AA) /\right.$ $\mathrm{Cu}(22 \AA)]_{8} / \mathrm{Cr}(20 \AA)$ в рамках теории эффективной среды по формулам Френеля и в приближении самоусреднения с учетом спин-зависящего рассеяния носителей в сравнении с экспериментальными данными по отражению и магнитоотражению, пропусканию и магнитопропусканию неполяризованного ИК-излучения приведены далее.

\section{4. Результаты расчета и сравнение с экспериментальными данными}

На рис. 3 представлена полевая зависимость магнитосопротивления $\Delta \rho / \rho$ для многослойной системы $\mathrm{Ni}_{48} \mathrm{Fe}_{12} \mathrm{Cr}_{40}(50 \AA) /\left[\mathrm{Co}_{90} \mathrm{Fe}_{10}(14 \AA) / \mathrm{Cu}(22 \AA)\right]_{8} / \mathrm{Cr}(20 \AA)$ при комнатной температуре. Магнитосопротивление демонстрирует резкий рост в малых полях и выходит на насыщение в поле $H>500$ Ое. Такое поведение является характерным для многослойных наноструктурированных систем $[1,2,8,16]$ и связано с изменением величины спин-зависящего рассеяния электронов проводимости при изменении магнитного порядка (направления намагниченности слоев) в наноструктуре под действием внешнего магнитного поля.

На рис. 3 указана полная амплитуда изменения магнитосопротивления $\left|\Delta \rho / \rho_{0}\right|=20.4 \%$ и величина отрицательного магнитосопротивления $\Delta \rho / \rho_{0}=15.8 \%$. В виду того, что в магнитооптических исследованиях сравнива-

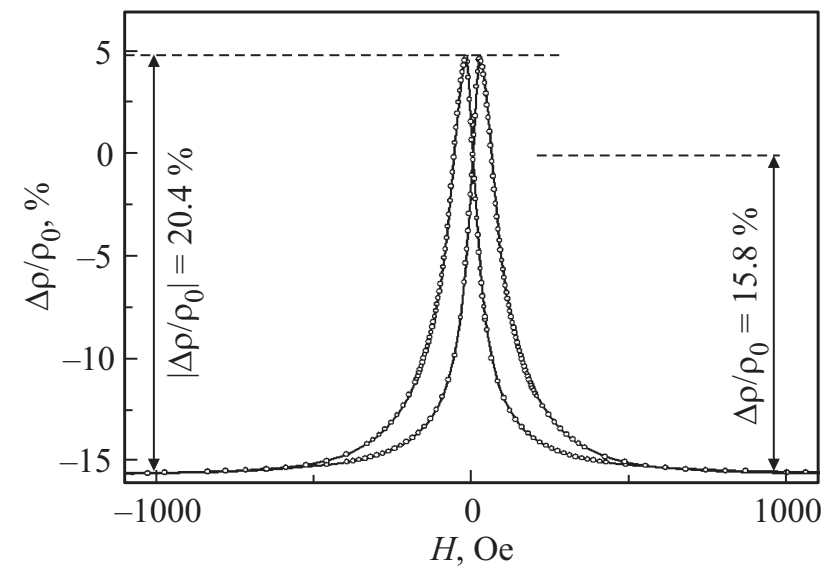

Рис. 3. Полевая зависимость магнитосопротивления $\left(\Delta \rho / \rho_{0}\right)$ для $\quad \mathrm{Ni}_{48} \mathrm{Fe}_{12} \mathrm{Cr}_{40}(50 \AA) /\left[\mathrm{Co}_{90} \mathrm{Fe}_{10}(14 \AA) / \mathrm{Cu}(22 \AA)\right]_{8} / \mathrm{Cr}(20 \AA)$ при $T=293 \mathrm{~K}$.

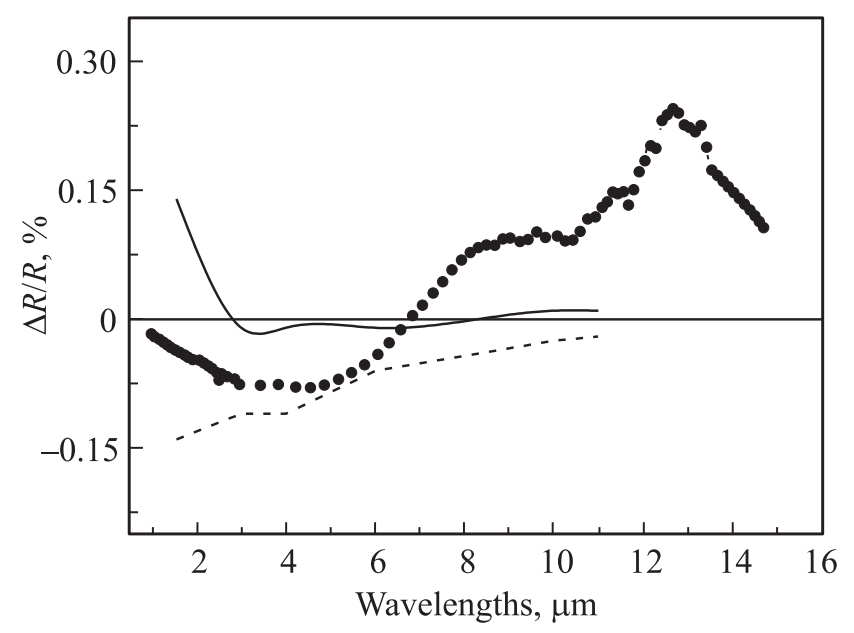

Рис. 4. Спектры МРЭ на отражении света $(\Delta R / R)$ для наноструктуры $\mathrm{Ni}_{48} \mathrm{Fe}_{12} \mathrm{Cr}_{40}(50 \AA) /\left[\mathrm{Co}_{90} \mathrm{Fe}_{10}(14 \AA) /\right.$ $\mathrm{Cu}(22 \AA)]_{8} / \mathrm{Cr}(20 \AA)$ : расчет по теории МРЭ (штриховая линия - по формулам Френеля, сплошная кривая - с учетом спин-зависящего рассеяния $\left.\left(\tau=9 \cdot 10^{-16} \mathrm{~s}, \beta=0.75[19]\right)\right)$ и экспериментальные данные $\Delta R / R$ (символы) при $T=293 \mathrm{~K}$, $H=3 \mathrm{kOe}(\mathbf{H} \| \mathbf{q}-$ плоскости структуры).

лись значения интенсивности отраженного (прошедшего) света от пленки, находящейся в нулевом внешнем магнитном поле и в поле насыщения, для расчета МРЭ была использована величина аналогичного изменения магнитосопротивления, т. е. $\Delta \rho / \rho_{0}=15.8 \%$. Надо отметить, что электросопротивление наноструктуры уменьшается при понижении температуры, при этом величина $\Delta \rho / \rho$ в поле насыщения меняется слабо.

На рис. 4 приведены расчетные и экспериментальный спектры МРЭ для многослойной наноструктуры для $\Delta \rho / \rho=15.8 \%$ при комнатной температуре. При общем подобии экспериментальных и расчетных кривых имеются различия. Видно, что в спектрах МРЭ значительную роль играет рассеяние (затухание) света на межслойных интерфейсах. Расчеты по формулам Френеля (7) дают 


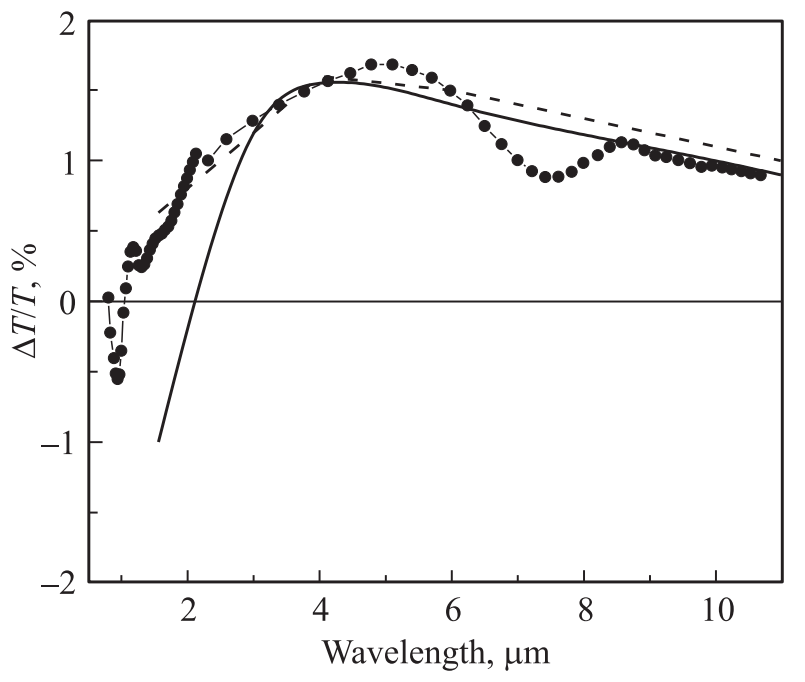

Pис. 5. Спектры МРЭ на пропускании света для многослойной наноструктуры $\mathrm{Ni}_{48} \mathrm{Fe}_{12} \mathrm{Cr}_{40}(50 \AA) /\left[\mathrm{Co}_{90} \mathrm{Fe}_{10}(14 \AA) /\right.$ $\mathrm{Cu}(22 \AA)]_{8} / \mathrm{Cr}(20 \AA)$ : расчетные (штриховая линия - по формулам Френеля, сплошная кривая - с учетом спин-зависящего рассеяния $\left.\left(\tau=9 \cdot 10^{-16} \mathrm{~s}, \beta=0.75[19]\right)\right)$ и экспериментальные данные $\Delta T / T$ (символы) при $T=293 \mathrm{~K}, H=7.5 \mathrm{kOe}$ (H $\| \mathbf{q}-$ плоскости структуры).

результаты близкие к экспериментальным (по интенсивности), однако не позволяют получить изменение знака МРЭ. Расчет по формуле (9) показывает, что смена знака МРЭ обусловлена значительным вкладом рассеяния на межслойных интерфейсах, что согласуется с литературными данными, например [21]. В общем случае, трудность получения количественного согласия заключается в том, что при учете многократного отражения света происходит существенная деформация и модуляция спектра $\Delta R / R$ в структуре.

На рис. 5 представлены расчетные и экспериментальный спектры МРЭ на пропускание света (магнитопропускание $\Delta T / T)$ при нормальном падении естественного света, комнатной температуре, $\Delta \rho / \rho=15.8 \%$ в поле насыщения. В отличие от МРЭ на отражении в случае магнитопропускания рассчитанные данные для обоих случаев хорошо совпадают с экспериментальными. Видно, что учет спин-зависящего рассеяния при тех же параметрах, что были применены для магнитоотражения, позволяет получить хорошее согласие с экспериментом в ИК-области спектра (рис. 5). Таким образом, только учет спин-зависящего рассеяния на интерфейсах позволяет описать смену знака МРЭ как на отражение, так и на прохождение света. Хорошее согласие с экспериментальными данными на пропускание позволяет предложить использованный метод расчета для качественной и количественной оценки МРЭ для металлических многослойных наноструктур на основе измеренного магнитосопротивления и табличных данных индексов рефракции.

Дополнительно можно отметить, что при комнатной температуре полученная экспериментально скорость из- менения величины магнитопропускания $\Delta T / T$ в области магнитных полей ниже поля насыщения достигает заметной величины $\sim 0.5 \% / 100$ Ое (на рисунке не показано), сравнимой с данными для других пленочных магнитных структур (см. работы $[13,21,22]$ и ссылки в них). Теоретические данные подтверждают возможность получения еще больших значений МРЭ для подобных структур [5], и экспериментальные работы в данном направлении необходимо продолжать. Данный результат исследований является важным для возможного прикладного применения металлических наноструктур, например, в качестве бесконтактного ИК-прозрачного сенсора магнитных полей или „оптических“ спиновых клапанов.

\section{5. Заключение}

В работе рассчитаны и измерены спектры магниторефрактивного эффекта на отражении (магнитоотражение) и пропускании (магнитопропускание) естественного света для металлической многослойной наноструктуры $\mathrm{Ni}_{48} \mathrm{Fe}_{12} \mathrm{Cr}_{40}(50 \AA) /\left[\mathrm{Co}_{90} \mathrm{Fe}_{10}(14 \AA) / \mathrm{Cu}(22 \AA)\right]_{8} / \mathrm{Cr}(20 \AA)$ в ИК-области спектра. Показано, что величина и вид спектральной зависимости магниторефрактивного эффекта зависят от величины магнитосопротивления (магнитопроводимости) и оптических параметров слоев многослойных наноструктур.

Предложенные формулы коэффициентов рефракции и экстинкции слоев наноструктуры в магнитном поле позволяют рассчитать значения магниторефрактивного эффекта на пропускании света, которые хорошо согласуются с экспериментальными данными. Спин-зависящее рассеяние электронов на интерфейсах слоев наноструктуры приводит к смене знака магниторефрактивного эффекта в области длин волн меньше $6 \mu \mathrm{m}$. Учет интерференции и поглощения света в слоях структуры по формулам Френеля без учета спин-зависящего рассеяния дает только качественное согласие с экспериментом.

Заметная величина магниторефрактивного эффекта (до $1.5 \%$ в поле $3.5 \mathrm{kOе}$ в широкой ИК-области) перспективна для развития методов бесконтактного экспрессисследования металлических пленочных наноструктур с эффектом гигантского магнитосопротивления. Результаты работы также могут найти применение как для дальнейшего развития теории МРЭ, так и для поиска новых перспективных материалов со значительными магнитооптическими и транспортными явлениями, которые играют важную роль в элементах современной электроники.

\section{Список литературы}

[1] S. Tumanski. Thin Film Magnetoresistive Sensors. Institute of Physics Publishing, Bristol-Philadelphia (2001). 441p.

[2] C. Reig, S. Cardoso de Freitas, S.C. Mukhopadhyay. Giant Magnetoresistance (GMR) Sensors. From Basis to State-of the-Art Applications. In Series: Smart Sensors, Measurement and Instrumentation. V. 6. Springer-Verlag, Berlin, Heidelberg (2013). 301 p. 
[3] M. McCormack, S. Jin, T.H. Tiefel, R.M. Fleming, J.M. Phillips, R. Ramesh. Appl. Phys. Lett. 64, 3045 (1994).

[4] E.L. Nagaev. Colossal Magnetoresistance and Phase Separation in Magnetic Semiconductors. Imperial College Press, London (2002). $457 \mathrm{p}$.

[5] J.C. Jacquet, T. Valet. In Series: Proceedings of the Materials Research Society Magnetic Ultrathin Films, Multilayer and Surfaces / Ed. E. Marinero. Materials Research Society, Pittsburg (1995) $477 \mathrm{p}$.

[6] A.V. Telegin, Yu.P. Sukhorukov, N.N. Loshkareva, E.V. Mostovshchikiva, N.G. Bebenin, E.A. Gan'shina, A.B. Granovsky. JMMM 383, 104 (2015).

[7] V.G. Kravets, D. Bozec, J.A.D. Matthew, S.M. Thompson. Phys. Rev. B 65, 054415 (2002).

[8] I.D. Lobov, M.M. Kirilova, A.A. Makhnev, L.N. Romashev, V.V. Ustinov. Phys. Rev. B 81, 134436 (2010).

[9] L.A. Bulavin, V.G. Kravets, K.L. Vinnichenko, D.Yu. Man'ko. J. Appl. Spectroscop. 68, 783 (2001).

[10] R.T. Mennicke, D. Bozec, V.G. Kravets, M. Vopsaroiu, J.A.D. Matthew, S.M. Thompson. JMMM 303, 92 (2006).

[11] А. Грановский, И. Быков, Е. Ганьшина, А. Козлов, А. Юрасов, Ю. Калинин, М. Инуе. ЖЭТФ 123, 1256 (2003).

[12] А.Б. Грановский, Е.А. Ганьшина, А.Н. Юрасов, Ю.В. Борискина, С.Г. Ерохин, А.Б. Ханикаев, М. Инуе, А.П. Виноградов, Ю.П. Сухоруков. Радиотехника и электроника 52, 1152 (2007).

[13] A. Granovsky, Yu. Sukhorukov, E. Gan'shina, A. Telegin. In: Springer Series in Materials Science. Magnetophotonics. From Theory to Applications V. 178 / Ed. M. Inoue, M. Levy, A.V. Baryshev. Springer, Berlin, Heidelberg (2013), P. 107.

[14] R.J. Baxter, D.G. Pettifir, E.Y. Tsymbal, D. Bozec, J. A.D. Matthew, S.M. Thompson. J. Phys. Condens. Matter. 15, L695 (2003).

[15] А.Н. Юрасов, А.В. Телегин, Ю.П. Сухоруков. ФТТ 58, 656 (2016).

[16] Н.С. Банникова, М.А. Миляев, Л.И. Наумова, В.В. Проглядо, Т.П. Криницина, И.Ю. Каменский, В.В. Устинов. ФММ 116, 1040 (2015).

[17] А.Н. Юрасов. Материаловедение 6, 32 (2014).

[18] В.М. Маевский. ФММ 59, 213 (1985).

[19] J.P. Camplin, S.M. Thompson, D.R. Loraine, D.I. Pugh, J.F. Collingwood. J. Appl. Phys. 87, 4846 (2000).

[20] В.В.Устинов, Ю.П. Сухоруков, М.А. Миляев, А.Б. Грановский, А.Н. Юрасов, Е.А. Ганьшина, А.В. Телегин. ЖЭТФ 135, 293 (2009).

[21] R.T. Mennicke, J.A.D. Matthew, S.M. Thompson. Rev. Sci. Instrum. 79, 023901 (2008).

[22] А.Н. Юрасов. РТЖ 1, 25 (2016). 